\title{
RASSEGNA
}

\section{Le sindromi ipereosinofile}

\author{
Hypereosinophilic syndromes
}

\section{Giuseppe Civardi*, Luca Zanlari, Emanuele Bassi, Roberta Bonassi, Corrado Ajolfi}

\author{
UOC Medicina Interna, Presidio Ospedaliero della Val d'Arda, Fiorenzuola d'Arda (PC), AUSL di Piacenza
}

Ricevuto il 21 aprile 2009; accettato il 28 aprile 2009

disponibile online il 13 luglio 2009

\section{KEYWORDS \\ Hypereosinophilic \\ syndrome; \\ FIP1L1-PDGFRA \\ fusion gene; \\ Imatinib; \\ Interleukin 5.}

\begin{abstract}
Summary
Background: The last few years have seen a complete change in the etiopathogenetic features, classification and therapeutic approach of the hypereosinophilic syndrome (HES), a multiorgan targeted blood disease. The discovery of a genetic mutation and the occurrence of a new fusion gene, named FIP1L1-PDGFRA (FIP gene), in some patients allowed the identification of a new myeloproliferative disorder, $M$-HES: thereafter, the pivotal therapeutic role of the tyrosine kinase inhibitors, particularly, imatinib mesylate, was clearly detected. In the same period a new pathogenetic mechanism has been detected: some authors described the presence of a CD3-CD4 + Tcell clone correlating with the overproduction of IL5, a potent eosinophilic cell line stimulating cytokine. As a consequence an international consensus committee proposed a new classification for these syndromes, in accordance with these new pathogenetic features. The disease is characterized by an extensive tissue and organ damage due to an eosinophilic cell infiltration and leading to the release of toxic cytokines and subsequent organ dysfunction. The heart, lungs, gastrointestinal apparatus, skin and central nervous system are affected. Moreover the released cytokines can induce a thrombophilic status and thromboembolic events can occur throughout the body.

Aim of the study: We describe the diagnostic procedures that are necessary in order to obtain a correct diagnosis and classification of the disease and to evaluate the presence of an organ and tissue damage. In particular, bone marrow biopsy and cytogenetic examination of blood and marrow are necessary for detecting M-HES cases that are positive for the FIP gene. In these patients, imatinib mesylate has a leading role for obtaining complete remission of the disease in a high percentage of cases. We also examine the therapeutic options for the other forms of the disease: prednisone, interferon, hydroxiurea are effective therapeutic tools in these patients. Finally, the new therapeutic perspectives, such as monoclonal antibodies directed against IL 5 or IL 5 receptor and novel tyrosine kinase inhibiting drugs, are examined.

(c) 2009 Elsevier Srl. All rights reserved.
\end{abstract}

\footnotetext{
* Corrispondenza: UOC Medicina Interna, Presidio Ospedaliero della Val d’Arda, via Roma 8 - 29017 Fiorenzuola d'Arda, PC.
} E-mail: g.civardi@ausl.pc.it (G. Civardi). 


\section{Introduzione}

Il concetto di sindrome ipereosinofila (HES), introdotto da Hardy e Anderson nel 1968 [1], è andato via via estendendosi e complicandosi nel tempo, in accordo con la scoperta di nuovi e diversi meccanismi patogenetici. Un primo punto cardine fu l'individuazione, negli anni Novanta [2,3], di forme a genesi linfoide, dovute a un'abnorme proliferazione linfocitaria $\mathrm{T}$ con anomala produzione di interleuchina 5 (IL5), potente induttore della proliferazione e differenziazione degli eosinofili. Negli anni successivi, la segnalazione dell'efficacia in alcune forme dell'imatinib mesilato [4,5], farmaco inibitore delle tirosinchinasi utilizzato con grande successo nella leucemia mieloide cronica, indusse alla scoperta in un discreto gruppo di pazienti di una traslocazione $4 q 12$, che generava un nuovo gene denominato FIP1L1PDGFRA (o più semplicemente FIP) il cui trascritto (una tirosinchinasi) era il bersaglio del farmaco $[6,7]$.

Questi nuovi dati resero ragione della notevole disomogeneità [8] anche clinica, oltre che patogenetica, delle diverse forme che richiesero da allora una classificazione sempre più complessa e articolata.

Scopo del presente articolo è fornire una rapida ed essenziale sistematizzazione di questo insieme disomogeneo di sindromi, finalizzata a suggerire un percorso diagnostico e terapeutico che possa fungere da guida nella pratica clinica quotidiana.

\section{Definizione, epidemiologia ed eziopatogenesi}

\section{Definizione}

I criteri diagnostici attualmente utilizzati per la diagnosi di HES sono stati definiti da almeno vent'anni [9] e continuano a mantenere la loro validità. Essi sono riassumibili in tre punti: - conta degli eosinofili $>1.500 / \mathrm{mm}^{3}$ per un periodo di almeno 6 mesi, oppure per un periodo più breve ma in presenza di danno d'organo accertato;

- assenza di evidenza di cause allergiche, parassitarie o acquisite di altra natura per la genesi dell'eosinofilia;

- segni e sintomi di coinvolgimento di organi o apparati.

\section{Epidemiologia}

Non sono disponibili in letteratura dati definiti sull'incidenza e prevalenza della sindrome nelle varie popolazioni; si tratta comunque di una patologia rara e verosimilmente sottodiagnosticata a causa delle caratteristiche cliniche e bioumorali piuttosto sfumate. Una stima approssimativa effettuata sulle diagnosi di dimissione suggerisce una prevalenza che equivale a circa un terzo di quella della leucemia mieloide cronica. La HES colpisce prevalentemente il sesso maschile, con una proporzione che varia da 4 a 9: 1 [10], e ha un'incidenza massima nelle classi di età da 20 a 50 anni, anche se può essere diagnosticata in tutte le fasce di età. L'identificazione di sottotipi di HES a patogenesi diversa ha poi portato alla dimostrazione che la distribuzione della patologia tra $i$ sessi non è uniforme: infatti, mentre nella forma mieloproliferativa predomina il sesso maschile, nella forma linfocitaria la prevalenza è la medesima nei due sessi [10].
È verosimile che un'applicazione rigorosa di un protocollo diagnostico mirato potrebbe portare alla luce un'incidenza indubbiamente più elevata, dato il numero notevole e sempre crescente di forme cliniche fruste o paucisintomatiche.

\section{Eziopatogenesi}

La proliferazione e differenziazione degli eosinofili avvengono da progenitori mieloidi per l'azione di alcuni fattori di crescita (GM-CSF, IL3, IL5) che agiscono sia come stimolanti della proliferazione e della differenziazione sia come inibitori dell'apoptosi [11]. La principale fonte di produzione di queste citochine è rappresentata da una sottoclasse linfocitaria T-helper di tipo 2 [12].

Due sono i principali meccanismi patogenetici noti che possono portare all'incontrollata proliferazione eosinofila e al conseguente danno d'organo secondario:

- il primo è legato a una mutazione a livello della cellula staminale emopoietica che determina una progressiva espansione clonale dei progenitori mieloidi, i quali tendono a una differenziazione preferenziale in senso eosinofilo;

- il secondo è legato a un'espansione eccessiva della linea linfocitaria $\mathrm{T}$ in grado di produrre una quantità troppo elevata di citochine che agiscono in senso proliferativo, differenziativo e antiapoptotico sulla linea eosinofila, determinando l'espressività clinica della malattia [13].

Come già accennato [6], del tutto recentemente il primo meccanismo patognetico ha ricevuto una chiara spiegazione molecolare con la scoperta di un'anomalia citogenetica "criptica", non individuabile cioè con le comuni metodiche di citogenetica. Una delezione interstiziale a livello del cromosoma 4q12 determina il giustapporsi di due geni, FIP1L1 e PDGFRA, a formare un nuovo gene di fusione denominato FIP, il cui trascritto è una proteina a potente attività tirosinchinasica. Quest'ultima determina una trasformazione in senso neoplastico della cellula staminale, che acquisisce capacità proliferative evidenziabili anche in vitro $e$ in assenza degli usuali fattori di crescita.

Tale proteina enzimatica dimostra, in vitro, una sensibilità all'attività di imatinib mesilato, potente inibitore dell'attività tirosinchinasica, 100 volte superiore a quella che il farmaco esercita a livello della proteina BCR-ABL, che ha un ruolo chiave nella patogenesi della leucemia mieloide cronica. Proprio dall'osservazione empirica dell'efficacia di questo farmaco in molti casi di HES ha preso avvio il filone di ricerca che ha portato ai risultati sopra citati. Secondo diversi autori, la frequenza con cui la mutazione verrebbe rilevata nei pazienti con HES si collocherebbe tra il $17 \%$ e il $56 \%$ [6]. Una parte di questi casi, inoltre, mostra, in associazione all'eosinofilia, anche un incremento dei mastociti fin quasi ad arrivare a quadri clinici "overlap" con la mastocitosi sistemica: tali pazienti presentano alcune caratteristiche cliniche distintive come splenomegalia, fibrosi endomiocardica, elevati livelli ematici di vitamina B12 e di triptasi.

Il gene di fusione denominato FIP1 (FIP1L1-PDGFRA), tuttavia, non è l'unica causa possibile della malattia: infatti, mentre il breakpoint di PDGFR è fisso e ristretto all'esone 12, la combinazione con un altro gene può essere variabile. Di recente sono stati decritti geni di fusione con BCR, K1F5B e CDK5RAP2, con un'espressione clinica del tutto simile alla forma classica $[14,15]$. 
Inoltre, sono stati descritti riarrangiamenti di altri geni, come PDGFRB o FGFR1 [16]: riarrangiamenti di quest'ultimo gene caratterizzano una forma atipica di sindrome mieloproliferativa che si presenta con eosinofilia, denominata leucemia/linfoma a cellule staminali, rara e a prognosi particolarmente severa [17].

Un secondo meccanismo patogenetico dimostrato in corso di HES riguarda la proliferazione di una linea linfocitaria $T$ aberrante, che esprime marcatori di superficie in modo anomalo (CD3-, CD4+) e determina un'iperproduzione di citochine quali GM-CSF, IL5, IL4 e IL13 [3]: l'effetto di questa sovrapproduzione di citochine è uno stimolo incontrollato alla proliferazione policlonale degli eosinofili. Collateralmente, si verifica anche una stimolazione cronica della linea linfocitaria B che comporta iperproduzione di lgE e ipergammaglobulinemia policlonale; sono state dimostrate anche anomalie di tipo clonale a livello dei linfociti T come anomalie citogenetiche ricorrenti. Pur trattandosi all'inizio di patologie benigne, tali forme sfociano con una certa frequenza in un linfoma a cellule T [18].

Questo tipo di meccanismo patogenetico è individuabile in circa il 20-25\% di casi di HES [8]. Pertanto, tenendo presente quanto esposto in precedenza, una buona metà dei casi di HES rimane non spiegata e viene classificata come forma idiopatica.

Nelgi ultimi anni, l'accumularsi di nuove osservazioni cliniche e di nuovi dati patogenetici ha ulteriormente arricchito, ma anche complicato, lo spettro nosografico della HES [19].

Sono state identificate forme di tipo familiare, sovente con espressione clinica minima o nulla, e forme di sovrapposizione con infiltrazione eosinofila limitata a organi o apparati ad andamento clinico variabile. Infine, nella nosografia della malattia sono state incluse anche condizioni di eosinofilia associate ad altre patologie, come la malattia di Churg-Strauss o la mastocitosi sistemica.

Nel 2005, un gruppo di lavoro della International Eosinophil Society ha definito una nuova classificazione onnicomprensiva, che è stata pubblicata l'anno successivo [20]. La tabella 1 riporta in modo sintetico tale classificazione.

\section{Clinica}

Le manifestazioni cliniche della HES sono generalmente proteiformi e legate parzialmente al sottotipo di malattia. Lo spettro di gravità dei paziente è ampio, variando da soggetti pressoché asintomatici sino a pazienti in condizioni critiche con compromissione di diversi organi e apparati e sintomi sistemici. Nella tabella 2 [8] sono riassunti i fondamentali dati clinici di raffronto tra i principali sottotipi di HES.

Oltre che alla compromissione sistemica (febbre, calo ponderale, prurito, sudorazione notturna, astenia) il quadro clinico è determinato dalla compromissione d'organo associata all'infiltrazione da parte della popolazione eosinofila: il danno tissutale è mediato da citochine proinfiammatorie e tossiche prodotte dalle cellule, come la proteina cationica $(E C P)$, la proteina basica maggiore (MBP) e altre ad azione enzimatica quali ribonucleasi (EDN) e perossidasi (EPC) [11]. A ciò si aggiungono una notevole produzione di radicali liberi, leucotrieni e prostaglandine e l'effetto fibrogenico dovuto alla liberazione di TGF-beta. Si verifica pertanto un danno necrotico tissutale su base infiammatoria seguito da fibrosi cicatriziale che determina un ulteriore danno funzionale. Tale processo necroinfiammatorio genera poi a livello tissutale uno stato protrombotico che può avere ricadute anche rilevanti sulla sintomatologia clinica.

\section{Tabella 1 Classificazione aggiornata delle sindromi ipereosinofile (HES) secondo l'International Eosinophil Society.}

\begin{tabular}{|c|c|c|}
\hline \multirow[t]{2}{*}{$\begin{array}{l}\text { HES mieloproliferativa } \\
\text { FIP1L1-PDGFRA }\end{array}$} & FIP1L1-PDGFRA positiva & $\begin{array}{l}\text { Presenza di anormalità citogenetiche e blastomi } \\
\text { Può evolvere verso leucemia cronica eosinofila }\end{array}$ \\
\hline & FIP1L1-PDGFRA negativa & $\begin{array}{l}\text { Eziologia sconosciuta Eosinofilia clonale associata a } 4 \text { o più dei } \\
\text { seguenti sdegni: } \\
\text { - displasia eosinofila } \\
\text { - anemia } \\
\text { - elevata vitamina B12 } \\
\text { - elevata triptasi } \\
\text { - piastrinopenia } \\
\text { - epatosplenomegalia } \\
\text { - ipercellularità midollare e/o fibrosi midollare }\end{array}$ \\
\hline HES linfocitica & \multicolumn{2}{|c|}{ Presenza di cloni cellulari T (CD3-, CD4+) producenti IL5 } \\
\hline \multirow[t]{3}{*}{ HES idiopatica } & Benigna & Assenza di coinvolgimento d'organo \\
\hline & Complessa & $\begin{array}{l}\text { Disfunzione d'organo in assenza di malattia mieloproliferativa o } \\
\text { linfocitaria }\end{array}$ \\
\hline & Episodica & Angioedema ciclico con eosinofilia \\
\hline HES familiare & \multicolumn{2}{|c|}{ Storia familiare documentata di eosinofilia persistente a causa sconosciuta } \\
\hline HES associate & \multicolumn{2}{|c|}{$\begin{array}{l}\text { Associata a diagnosi accertata di infezione da HIV, malattia di Churg-Strauss, sarcoidosi, mastocitos } \\
\text { sistemica, malattia infiammatoria intestinale ecc. }\end{array}$} \\
\hline
\end{tabular}

HES "overlap"

Associata a malattie eosinofile d'organo: eosinofilia gastrointestinale, polmonite eosinofila, sindrome eosinofilia-mialgia

Fonte: Klion AD, et al. J Allergy Clin Immunol 2006;117(6):1292-302. 
Tabella 2 Sindrome ipereosinofila (HES): principali dati clinici e fisiopatologici.

\begin{tabular}{|c|c|c|c|}
\hline Sottotipo & Mieloproliferativa & Linfoide & Idiopatica \\
\hline \multirow[t]{4}{*}{$\begin{array}{l}\text { Fisiopatologia } \\
\text { e dati clinici }\end{array}$} & $\begin{array}{l}\text { Presenza di mutazione } \\
\text { FIP1L1-PDGFRA o altre } \\
\text { mutazioni similari }\end{array}$ & $\begin{array}{l}\text { Clone } \mathrm{T} \text { linfocitario } \mathrm{TH} 2 \\
\text { producente citochine (IL5). }\end{array}$ & $\begin{array}{l}\text { Vasculite necrotizzante } \\
\text { eosinofila }\end{array}$ \\
\hline & $\begin{array}{l}\text { HES "classica": maschi con } \\
\text { malattia endomiocardica; } \\
\text { ipercellularità midollare con } \\
\text { aumento delle mastcellule }\end{array}$ & $\begin{array}{l}\text { Cloni linfocitari T sporadicamente } \\
\text { presenti anche nella malattia di } \\
\text { Churg-Strauss, EAE e sindrome } \\
\text { NERDS }\end{array}$ & $\begin{array}{l}\text { Angioedema episodico con } \\
\text { eosinofilia (EAE), sindrome } \\
\text { NERDS }\end{array}$ \\
\hline & $\begin{array}{l}\text { Ulcere mucosali: quadro clinico } \\
\text { impegnativo, legato alle } \\
\text { manifestazioni cardiache; } \\
\text { prognosi più severa }\end{array}$ & $\begin{array}{l}\text { Non vi sono differenze di sesso: } \\
\text { prevalenti manifestazioni cutanee, } \\
\text { prognosi più favorevole, possibile } \\
\text { evoluzione in linfoma T periferico }\end{array}$ & $\begin{array}{l}\text { Varietà di manifestazioni } \\
\text { cliniche: lesioni cutanee, } \\
\text { vasculite, patologia } \\
\text { immunologica, gastroenterite } \\
\text { eosinofila }\end{array}$ \\
\hline & $\begin{array}{l}\text { Elevati livelli di triptasi e } \\
\text { vitamina B12, fibrosi tissutale, } \\
\text { organomegalia con incremento } \\
\text { midollare di mastociti } \\
\text { fusiformi CD25+ }\end{array}$ & & \\
\hline Terapia & $\begin{array}{l}\text { Imatinib mesilato terapia di scelta. } \\
\text { Alcuni pazienti possono rispondere } \\
\text { all'interferone o ai monoclonali } \\
\text { anti-IL5 (mepolizumab) }\end{array}$ & $\begin{array}{l}\text { La maggioranza dei pazienti } \\
\text { risponde al cortisone. Dati } \\
\text { preliminari incoraggianti con gli } \\
\text { anti-IL5. Utile idrossiurea. } \\
\text { Interferone da utilizzare con cautela }\end{array}$ & $\begin{array}{l}\text { Fondamentale il controllo } \\
\text { dell'eosinofilia, correlata } \\
\text { al danno d'organo: steroidi, } \\
\text { idrossiurea, interferone }\end{array}$ \\
\hline
\end{tabular}

Legenda: EAE = angioedema episodico con eosinofilia; NERDS = Nodules, Eosinophilia, Rheumatism, Dermatitis and Swelling.

Gli organi e apparati colpiti più di frequente sono la cute, il cuore, l'apparato respiratorio, il sistema nervoso centrale e periferico e l'apparato gastroenterico. Sono inoltre presenti importanti alterazioni di tipo ematologico e coagulativo [13].

\section{Cute e annessi}

Sono presenti con notevole frequenza manifestazioni a tipo orticaria e angioedema, eritema e papule nodulari molto pruriginose. Le manifestazioni cutanee sono più caratteristiche della HES a genesi linfocitaria (assumendo talora la forma di angioedema episodico con eosinofilia o sindrome di Gleich), mentre nella HES mieloproliferativa anche le mucose possono essere coinvolte.

\section{Cuore}

Il danno cardiaco si estrinseca tipicamente a livello della regione endomiocardica e può essere distinto in tre stadi: un primo stadio, cosiddetto "necrotico", che decorre generalmente asintomatico ma che talora può manifestarsi con scompenso cardiaco acuto. In questo stadio l'infiltrazione eosinofila determina necrosi a livello dell'endocardio delle camere cardiache. A ciò fa seguito lo stadio "trombotico", in cui le superfici endocardiche lesionate diventano sede di deposizioni trombotiche a livello sia degli atri sia dei ventricoli: tali deposizioni possono staccarsi determinando episodi embolici polmonari e sistemici. Infine il terzo stadio, denominato "fibrotico", è caratterizzato dalle alterazioni cicatriziali che determinano fibrosi endomiocardica con conseguente cardiomiopatia restrittiva e scompenso cardiaco da alterazioni della compliance ventricolare. Tali alterazioni sono più frequenti nella forma clonale della HES, sono irreversibili quando giunte allo stadio fibrotico e per questo prognosticamente rilevanti in senso negativo.

\section{Polmoni}

Le manifestazioni cliniche a carico dell'apparato respiratorio mostrano un'ampia gamma di sintomi a vari livelli: si va da una sintomatologia prevalentemente a carico dell'albero tracheo-bronchiale (con tosse secca e stizzosa persistente, broncospasmo, dispnea da sforzo e generici sintomi di iperreattività bronchiale) alla comparsa di infiltrati polmonari multipli e migranti, sino alla pneumopatia restrittiva con fibrosi polmonare anche grave nelle forme più avanzate. Le manifestazioni a carico dell'interstizio polmonare sono più severe e frequenti nelle forme F/P positive.

\section{Sistema nervoso}

Può essere coinvolto il sistema nervoso centrale come il periferico: nel primo caso la sintomatologia può mimare quella di una sindrome a tipo demenza con confusione mentale, perdita della memoria, alterazioni comportamentali, deficit cognitivo; nel secondo caso prevale la sintomatologia sensitivo motoria, con quadro di polineuropatia con deficit sia simmetrici che asimmetrici. Inoltre, lo stato trombofilico può determinare quadri clinici di stroke o ischemia cerebrale transitoria. La sintomatologia neurologica è solitamente assente nelle forme di tipo linfocitario, mentre è presente sia in quelle di tipo idiopatico sia nelle forme F/P positive. 


\section{Apparato gastroenterico}

La sintomatologia digestiva è essenzialmente associata all'infiltrazione parietale del tubo gastroenterico da parte della popolazione eosinofila, con comparsa di dolori addominali, diarrea, dispepsia, nausea, epigastralgie. Il quadro clinico è descritto in dettaglio nel case-report pubblicato nel presente numero della rivista, al quale si rimanda.

\section{Sistema emocoagulativo}

L'ipereosinofilia causa danni alla superficie endovascolare, producendo vasculopatie periferiche e inducendo uno stato protrombotico: tali manifestazioni si estrinsecano principalmente a carico dei distretti vascolari più periferici, come le dita delle mani e dei piedi, causando fenomeni ischemici e necrotici.

\section{Procedure diagnostiche}

Una volta identificata l'ipereosinofilia ed esclusa una forma secondaria, se il paziente risponde ai criteri diagnostici generali prima elencati, si impone una più approfondita valutazione [21] allo scopo di:

- confermare la diagnosi;

- inquadrare il paziente nei vari sottotipi;

- effettuare una valutazione del danno d'organo.

Tutto ciò va effettuato ai fini di una terapia mirata in senso fisiopatologico, la quale può avvalersi oggi, come vedremo, di approcci molto efficaci e innovativi.

La raccolta di un'anamnesi accurata è importante nell'escludere forme secondarie (infezioni parassitarie, storia di allergia ecc.), mentre può indirizzare verso una forma familiare se il riscontro dell'ipereosinofilia è ricorrente tra $i$ parenti del paziente.

L'esame obiettivo permette di effettuare una prima rilevazione dei danni d'organo, rilevando la presenza di lesioni cutanee, di epatosplenomegalia, linfoadenopatie o di anormalità auscultatorie a livello cardiaco.

La valutazione degli esami bioumorali deve essere indirizzata alla ricerca ed esclusione di cause primarie di eosinofilia (patologie parassitarie, oncoematologiche e di tipo disreattivo autoimmune). Infine, la dimostrazione di elevati livelli di triptasi e/o di vitamina B12 indirizzano verso una forma mieloproliferativa. Uno studio delle sottopopolazioni linfocitarie può permettere l'identificazione di un'espansione della popolazione linfoide TH2 (CD3-, CD4+) e orientare verso una ipereosinofilia di tipo linfocitario.

La biopsia midollare con valutazione citogenetica e ricerca dell'anomalia cromosomica FIP1L1-PDGFRA e di quelle a essa correlate è da considerarsi il test diagnostico cardine per le ipereosinofilie mieloproliferative, con importanti ricadute sul piano terapeutico, data la loro spiccata sensibilità alla terapia con inibitori delle tirosinchinasi.

La diagnostica per immagini dovrà innanzitutto prendere in considerazione i potenziale organi bersaglio della malattia, in primo luogo il cuore. È indispensabile, pertanto, l'esecuzione di un ecocardiogramma e di una TAC del torace e dell'addome.

La dimostrazione del danno d'organo legato all'ipereosinofilia richiede inoltre la biopsia di tutte le sedi tissutali accessibili nelle quali si sospetti un'infiltrazione eosinofila: in particolare, la presenza di sintomi anche sfumati a livello del tubo digerente va indagata con metodiche endoscopiche.

\section{Terapia}

La strategia terapeutica della HES è decisamente mutata negli ultimi anni grazie alle nuove scoperte sui meccanismi patogenetici, che hanno portato alla riclassificazione della malattia in categorie diverse e ben definite. In particolare, l'individuazione della mutazione genetica che sta alla radice del meccanismo patogenetico della HES mieloproliferativa ha consentito di raggiungere importanti risultati utilizzando i nuovi farmaci inibitori delle tirosinchinasi, la cui efficacia ha radicalmente modificato la prognosi della malattia. L'impiego dell'imatinib mesilato ha permesso di ottenere remissioni sia cliniche sia molecolari con la scomparsa almeno temporanea del clone cellulare malato anche allo studio di citogenetica midollare. Inoltre, per le altre forme di malattia, diversi presidi terapeutici (interferone, anticorpi monoclonali anti-IL5 e anti-CD52) sono andati ad aggiungersi alla classica terapia steroidea o all'idrossiurea e anche per questi pazienti le prospettive curative sono migliorate.

\section{HES mieloproliferativa}

Per tutte le forme FIP1 positive, oggi la terapia di scelta è l'imatinib mesilato: nella stragrande maggioranza dei pazienti, il farmaco determina la pronta riduzione del numero di eosinofili e la regressione del danno d'organo associato alla malattia [4]. Nei pazienti con splenomegalia e danno polmonare, la percentuale di risposta supera il $90 \%$. Inoltre, la maggiore sensibilità del transcritto FIP1L1-PDGFRA rispetto al BCR-ABL fa sì che i dosaggi richiesti per l'ottenimento della risposta siano notevolmente più bassi nella HES [22] (100 mg/die come attacco e fino a $200 \mathrm{mg} /$ settimana come mantenimento) rispetto a quelli impiegati di routine nella leucemia mieloide cronica $(400-600 \mathrm{mg} / \mathrm{die})$ : ciò ha un'importante ricaduta positiva per quanto riguarda gli effetti collaterali legati al farmaco, notevolmente minori ai dosaggi somministrati nella HES. La risposta, inoltre, è rapida e si manifesta nell'arco di pochi giorni.

Particolare attenzione va peraltro posta nei pazienti con danno miocardico anche subclinico: la tossicità cardiaca dell'imatinib può infatti andare a sovrapporsi a quella dovuta alla liberazione rapida e massiva di sostanze cardiotossiche provenienti dagli eosinofili in disfacimento: sono stati descritti casi di scompenso cardiaco acuto subito dopo l'inizio della terapia [5,23]. Nei casi di danno endomiocardico, è indispensabile un controllo frequente e scrupoloso della troponina e dei parametri ecocardiografici durante la somministrazione con imatinib; il pretrattamento con prednisone potrebbe, secondo alcuni autori, ridurre la possibilità di tossicità cardiaca all'inizio del trattamento [23].

Riguardo alla durata della terapia, mancano ancora dati a lungo termine: è incerto, infatti, se gli inibitori delle tirosinchinasi possano essere in grado di guarire la malattia e quanto a lungo debbano essere somministrati per ottenere tale effetto. Nei casi segnalati di sospensione della terapia dopo scomparsa del marcatore molecolare, tuttavia, la sindrome 
ha mostrato tendenza alla recidiva con progressiva ricomparsa del clone mutato [24,25].

Sono stati poi segnalati casi di resistenza primaria alla terapia con imatinib in pazienti portatori di mutazioni puntiformi del gene diverse da quella classica (per esempio, T1949C o T2034C) [26].

Recentemente sono stati sintetizzati altri inibitori delle tirosinchinasi, come il sorafenib o il dasatinib, efficaci sia nella leucemia mieloide cronica sia nella HES. Il ruolo di questi nuovi farmaci nella strategia terapeutica della HES mieloproliferativa è tuttora da stabilire, Altri farmaci inibitori delle tirosinchinasi, ma con meccanismi d'azione addizionali (PKC412, EXEL-0862), sono in fase di studio e hanno fornito risultati promettenti in vitro o in animali da laboratorio [26].

La strategia terapeutica dei casi di HES mieloproliferativa senza marcatore citogenetico tipico è più complessa e aleatoria: nell'ipotesi che alcuni di questi soggetti fossero portatori di anomalie citogenetiche simili a quella classica, ma non evidenziabili con le normali tecniche di esame, sono stati compiuti diversi tentativi con l'inibitore delle tirosinchinasi: tali pazienti hanno presentato una risposta variabile e incostante all'imatinib e frequenti sono i risultati parziali o transitori. Nei casi refrattari alle terapie tradizionali con caratteristiche cliniche indirizzanti verso la forma mieloproliferativa, è comunque indicato un breve tentativo con imatinib a dosaggio più elevato $(400 \mathrm{mg} / \mathrm{die})$ da proseguirsi qualora i risultati clinici siano positivi. In questi pazienti sono stati compiuti tentativi con interferone da solo o associato a prednisone o idrossiurea, con risultati variabili ma talora incoraggianti (si veda oltre).

\section{HES linfocitaria}

La terapia steroidea rimane ancora il caposaldo nel trattamento di queste forme: il dosaggio iniziale è di $1 \mathrm{mg} / \mathrm{kg} / \mathrm{die}$ in equivalente prednisone. In tali casi, l'idrossiurea non ha fornito risultati apprezzabili, cosi come le altre terapie citotossiche (clorambucil, vincristina, ciclofosfamide) solitamente efficaci nelle malattie di tipo linfoproliferativo.

La terapia con interferone è stata associata da diversi autori [27] alla regressione, almeno parziale, del clone cellulare T CD3-CD4+: esistono, tuttavia, anche segnalazioni di indirizzo opposto secondo le quali l'interferone avrebbe determinato, in vitro, l'aumento della sopravvivenza del clone cellulare T inibendone l'apoptosi [28]. Si è suggerito, quindi, di associare all'interferone un farmaco, come il cortisone, in grado di stimolare l'apoptosi del clone cellulare T. I dosaggi richiesti sono generalmente bassi, attorno a 1-2 MU/die e la risposta compare lentamente, di solito dopo diverse settimane di terapia. Gli effetti collaterali sono quelli tipici della terapia con interferone (alopecia, depressione, sindrome similinfluenzale, induzione di patologie autoimmuni). I pazienti descritti in letteratura come responder all'interferone avevano caratteristiche cliniche compatibili in alcuni casi con HES mieloproliferativa e in altri casi con HS leucocitaria.

\section{Ulteriori possibilità terapeutiche}

Del tutto recentemente sono apparsi sulla scena terapeutica della HES [29] almeno due anticorpi monoclonali contro il recettore della IL5, il mepolizumab e il reslizumab (quest'ultimo oggi non più disponibile). In particolare, alcuni studi condotti a livello internazionale con mepolizumab $750 \mathrm{mg}$ ev una volta al mese [30] avrebbero dimostrato come nella HES tale farmaco sia in grado di ridurre in numero degli eosinofili limitando il danno tissutale a fronte di effetti collaterali minimi. In questi casi è stata dimostrata anche una caduta della proteina cationica correlata con il miglioramento clinico presentato dai pazienti. Entrambi i monoclonali avrebbero evidenziato un effetto sostanzialmente di risparmio sul dosaggio dello steroide, controllando la malattia anche con dosi di mantenimento più ridotte di quelle sopra segnalati. La risposta è comunque lenta e richiede settimane o mesi di trattamento; in molti casi, tuttavia, si è mantenuta a lungo anche dopo sospensione della terapia. Gli effetti positivi dei monoclonali anti-IL5 non correlerebbero né con il tipo di malattia né con la precedente risposta agli steroidi.

Segnalazioni sporadiche di buoni risultati in pazienti refrattari a diverse terapie sono stati riportati anche con l'alemtuzumab, monoclonale anti-CD52, antigene presente sulla superficie sia dei linfociti sia degli eosinofili, e con l'infliximab, monoclonale anti-interferone-alfa, quest'ultimo utilizzato con successo in un paziente con HES idiopatica e importante eritrodermia [31].

Infine, un'ulteriore opzione terapeutica in casi selezionati, particolarmente gravi e refrattari alle terapie convenzionali, può essere rappresentata dal trapianto allogenico di midollo osseo: ovviamente in questi casi s'impone un attento bilancio tra i rischi connessi con la procedura e quelli associati alla malattia. Candidati potenziali potrebbero trovarsi tra i pazienti affetti da HES mieloproliferativa refrattari alla terapia con inibitori delle tirosinchinasi o tra quelli con HES linfocitaria evoluta in linfoma T [20].

\section{Conclusioni}

La sindrome ipereosinofila, o meglio, le sindromi ipereosinofile rappresentano un campo in cui l'evoluzione delle conoscenze è particolarmente rapido: anche il tono a tratti interlocutorio di questa revisione ne risente in modo determinante. È verosimile che la classificazione qui esposta verrà ben presto rivista e modificata alla luce di nuove acquisizioni sui meccanismi patogenetici che sicuramente emergeranno nei prossimi anni: sempre più pazienti usciranno dalla categoria cosiddetta "idiopatica/complessa" per rientrare in categorie nosografiche più definite. Nello stesso tempo, sempre nuovi farmaci e approcci terapeutici stanno mutando in maniera significativa la prognosi dei soggetti con HES.

In questo continuo divenire, il punto di vista olistico proprio dell'internista appare un angolo d'osservazione privilegiato. Infatti, nel crocevia della HES convergono molteplici e diverse competenze: quella ematologica, quella immunopatologica e quella allergologica, senza trascurare che molti di questi pazienti presentano un danno d'organo che può giungere all'osservazione dello specialista (gastroenterologo, pneumologo, cardiologo ecc.). In un tale caleidoscopio di punti di vista parziali, è tutt'altro che semplice mantenere quello sguardo d'insieme che può consentire di cogliere una diagnosi a volte complessa, ma a volte semplice a patto di saper valutare il paziente a tutto tondo, con il suo 
mosaico complesso di sintomi, segni clinici e dati bioumorali e strumentali.

\section{Conflitto di interessi}

Gli autori dichiarano di essere esenti da conflitto di interessi.

\section{Bibliografia}

[1] Hardy WR, Anderson RE. The hypereosinophilic syndromes. Ann Intern Med 1968;68(6):1220-9.

[2] Cogan E, Schandené L, Crusiaux A, Cochaux P, Velu T, Goldman $M$. Brief report: clonal proliferation of type 2 helper T cells in a man with the hypereosinophilic syndrome. N Engl J Med 1994; 330(8):535-8.

[3] Simon HU, Plötz SG, Dummer R, Blaser K. Abnormal clones of T cells producing interleukin- 5 in idiopathic eosinophilia. N Engl J Med 1999;341(15):1112-20.

[4] Gleich GJ, Leiferman KM, Pardanani A, Tefferi A, Butterfield JH. Treatment of hypereosinophilic syndrome with imatinib mesilate. Lancet 2002;359(9317):1577-8.

[5] Pardanani A, Reeder T, Porrata LF, Ly CY, Baxter EJ, Cross NC, et al. Imatinib therapy for hypereosinophilic syndrome and other eosinophilic disorders. Blood 2003;101(9):3391-7.

[6] Cools J, DeAngelo DJ, Gotlib J, Stover EH, Legare RD, Cortes J, et al. A tyrosine kinase created by fusion of the PDGFRA and FIP1L1 genes as a therapeutic target of imatinib in idiopathic hypereosinophilic syndrome. N Engl J Med 2003;348(13):120114.

[7] Griffin JH, Leung J, Bruner RJ, Caligiuri MA, Briesewitz R. Discovery of a fusion kinase in EOL-1 cells and idiopathic hypereosinophilic syndrome. Proc Natl Acad Sci USA 2003; 100(13):7830-5.

[8] Gleich GJ, Leiferman KM. The hypereosinophilic syndromes: still more heterogeneity. Curr Opin Immunol 2005;17(6):679_ 84.

[9] Chusid MJ, Dale DC, West BC, Wolff SM. The hypereosinophilic syndrome: analysis of fourteen cases with review of the literature. Medicine (Baltimore) 1975;54(1):1-27.

[10] Weller PF, Bubley GJ. The idiopathic hypereosinophilic syndrome. Blood 1994;83(10):2759-79.

[11] Rothenberg ME, Hogan SP. The eosinophil. Annu Rev Immunol 2006;24:147-74.

[12] Romagnani S. Th1 and Th2 in human diseases. Clin Immunol Immunopathol 1996;80(3 Pt 1):225-35.

[13] Roufosse FE, Goldman M, Cogan E. Hypereosinophilic syndromes. Orphanet J Rare Dis 2007;2:37.

[14] Score J, Curtis C, Waghorn K, Stalder M, Jotterand M, Grand FH, et al. Identification of a novel imatinib responsive KIF5BPDGFRA fusion gene following screening for PDGFRA overexpression in patients with hypereosinophilia. Leukemia 2006; 20(5):827-32.

[15] Walz C, Curtis C, Schnittger S, Schultheis B, Metzgeroth G, Schoch $C$, et al. Transient response to imatinib in a chronic eosinophilic leukemia associated with ins $(9 ; 4)(q 33 ; q 12 q 25)$ and a CDK5RAP2-PDGFRA fusion gene. Genes Chromosomes Cancer 2006;45(10):950-6.

[16] Yamada Y, Rothenberg ME, Lee AW, Akei HS, Brandt EB, Williams DA, et al. The FIP1L1-PDGFRA fusion gene cooperates with IL-5 to induce murine hypereosinophilic syndrome (HES)/chronic eosinophilic leukemia (CEL)-like disease. Blood 2006;107(10): 4071-9.

[17] Macdonald D, Reiter A, Cross NC. The 8p11 myeloproliferative syndrome: a distinct clinical entity caused by constitutive activation of FGFR1. Acta Haematol 2002;107(2):101-7.

[18] de Lavareille A, Roufosse F, Schmid-Grendelmeier P, Roumier $A S$, Schandene $L$, Cogan $E$, et al. High serum thymus and activation-regulated chemokine levels in the lymphocytic variant of the hypereosinophilic syndrome. J Allergy Clin Immunol 2002;110(3):476-9.

[19] Gleich GJ, Leiferman KM. The hypereosinophilic syndromes: current concepts and treatments. Br J Haematol 2009;145(3): 271-85.

[20] Klion AD, Bochner BS, Gleich GJ, Nutman TB, Rothemberg ME, Simon HU, et al., The Hypereosinophilic Syndromes Working Group. Approaches to the treatment of hypereosinophilic syndromes: a workshop summary report. J Allergy Clin Immunol 2006;117(6):1292-302.

[21] Peros-Golubicić T, Smojver-Jezek S. Hypereosinophilic syndrome: diagnosis and treatment. Curr Opin Pulm Med 2007; 13(5):422-7.

[22] Helbig G, Stella-Hołowiecka B, Majewski M, Calbecka M, Gajkowska J, Klimkiewitcz R, et al. A single weekly dose of imatinib is sufficient to induce and maintain remission of chronic eosinophilic leukaemia in FIP1L1-PDGFRA-expressing patients. Br J Haematol 2008;141(2):200-4.

[23] Pitini V, Arrigo C, Azzarello D, La Gattuta G, Amata C, Righi M, et al. Serum concentration of cardiac troponin $T$ in patients with hypereosinophilic syndrome treated with imatinib is predictive of adverse outcomes. Blood 2003;102(9):3456-7.

[24] Jovanovic JV, Score J, Waghorn K, Cilloni D, Gottardi E, Metzegeroth G, et al. Low-dose imatinib mesylate leads to rapid induction of major molecular responses and achievement of complete molecular remission in FIP1L1-PDGFRA-positive chronic eosinophilic leukemia. Blood 2007;109(11):4635-40.

[25] Pardanani A, Ketterling RP, Li CY, Patnaik MM, Wolanskji AP, Eliot MA, et al. FIP1L1-PDGFRA in eosinophilic disorders: prevalence in routine clinical practice, long-term experience with imatinib therapy, and a critical review of the literature. Leuk Res 2006;30(8):965-70.

[26] Simon D, Salemi S, Yousefi S, Simon HU. Primary resistance to imatinib in Fip1-like 1-platelet-derived growth factor receptor alpha-positive eosinophilic leukemia. J Allergy Clin Immunol 2008;121(4):1054-6.

[27] Pan J, Quintás-Cardama A, Manshouri T, Giles FJ, Lamb P, Tefferi A, et al. The novel tyrosine kinase inhibitor EXEL-0862 induces apoptosis in human FIP1L1-PDGFR-alpha-expressing cells through caspase-3-mediated cleavage of Mcl-1. Leukemia 2007;21(7):1395-404.

[28] Schandené L, Roufosse F, de Lavareille A, Stordeur P, Efira A, Kennes $B$, et al. Interferon alpha prevents spontaneous apoptosis of clonal Th2 cells associated with chronic hypereosinophilia. Blood 2000;96(13):4285-92.

[29] Sutton SA, Assa'ad AH, Rothenberg ME. Anti-IL-5 and hypereosinophilic syndromes. Clin Immunol 2005;115(1):51-60.

[30] Plötz SG, Simon HU, Darsow U, Simon D, Vassina E, Yousefi S, et al. Use of an anti-interleukin- 5 antibody in the hypereosinophilic syndrome with eosinophilic dermatitis. N Engl J Med 2003;349(24):2334-9.

[31] Pitini V, Teti D, Arrigo C, Righi M. Alemtuzumab therapy for refractory idiopathic hypereosinophilic syndrome with abnormal T cells: a case report. Br J Haematol 2004;127(5):477. 